This item was submitted to Loughborough's Research Repository by the author.

Items in Figshare are protected by copyright, with all rights reserved, unless otherwise indicated.

\title{
Complex dynamic behaviors of nonequilibrium atmospheric dielectric-barrier
} discharges

PLEASE CITE THE PUBLISHED VERSION

PUBLISHER

(c) American Institute of Physics

VERSION

VoR (Version of Record)

LICENCE

CC BY-NC-ND 4.0

\section{REPOSITORY RECORD}

Zhang, Yuan Tao, De Zhen Wang, and Michael G. Kong. 2019. "Complex Dynamic Behaviors of Nonequilibrium Atmospheric Dielectric-barrier Discharges". figshare. https://hdl.handle.net/2134/5176. 
This item was submitted to Loughborough's Institutional Repository (https://dspace.lboro.ac.uk/) by the author and is made available under the following Creative Commons Licence conditions.

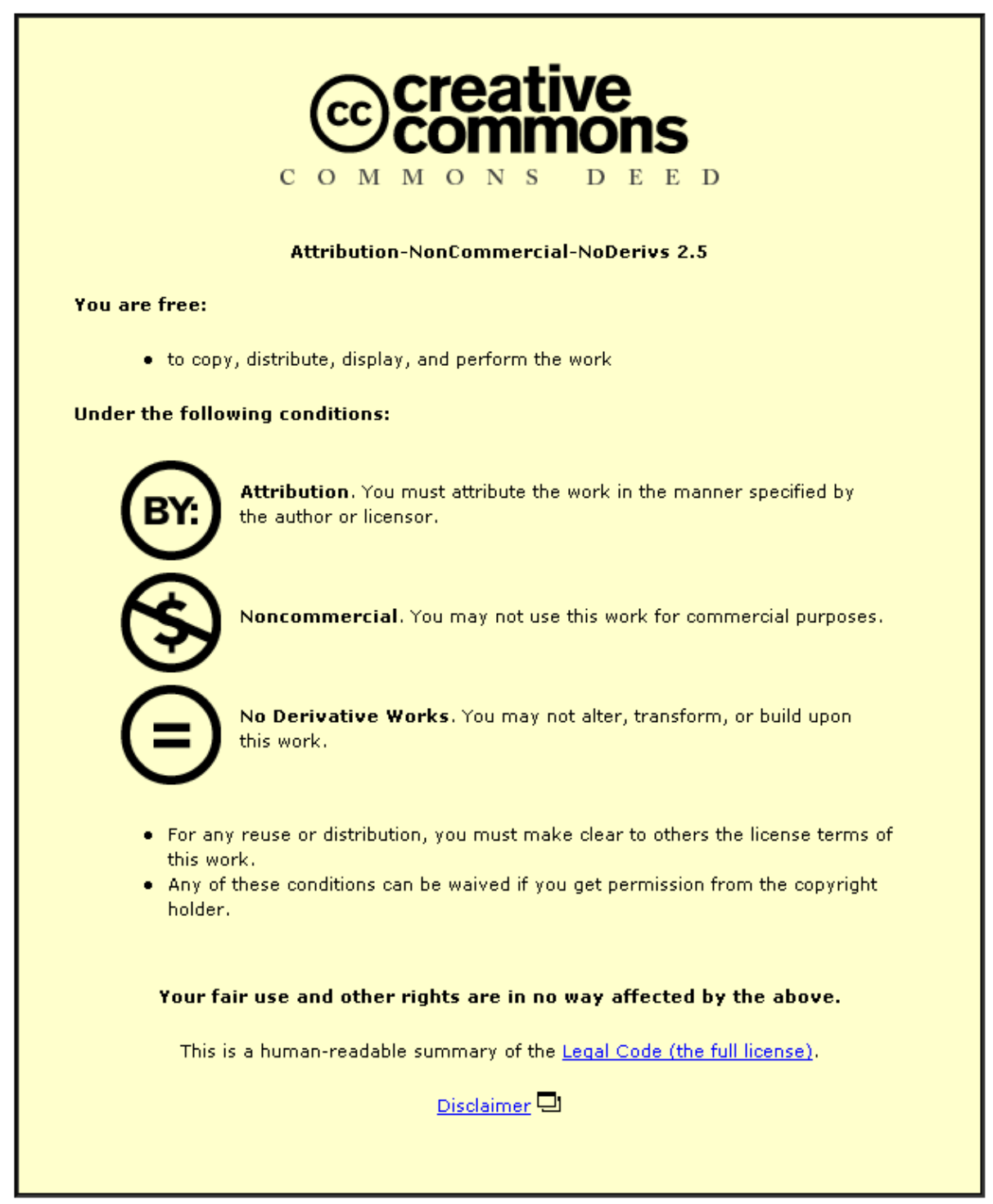

For the full text of this licence, please go to: http://creativecommons.org/licenses/by-nc-nd/2.5/ 


\title{
Complex dynamic behaviors of nonequilibrium atmospheric dielectric-barrier discharges
}

\author{
Yuan Tao Zhang and De Zhen Wang ${ }^{\text {a) }}$ \\ State Key Laboratory of Materials Modification by Laser, Ion and Electron Beams, Department of Physics, \\ Dalian University of Technology, Dalian 116024, People's Republic of China \\ M. G. Kong \\ Department of Electronic and Electrical Engineering, Loughborough University, Leics LE11 3TU, United \\ Kingdom
}

(Received 5 February 2006; accepted 8 July 2006; published online 22 September 2006)

\begin{abstract}
In this paper, a one-dimensional fluid model is used to investigate complex dynamic behaviors of a nonequilibrium dielectric-barrier discharge (DBD) in atmospheric helium. By projecting its evolution trajectory in the three-dimensional phase space of gas voltage, discharge current density, and electrode-surface charge density, the atmospheric DBD is shown to undergo a sequence of complex bifurcation processes when the applied voltage is increased from prebreakdown to many times of the breakdown voltage. Once the gas voltage exceeds the breakdown voltage, the discharge plasma is found to acquire negative differential conductivity and as a result its stability is compromised. For atmospheric DBD, however, the resulting low plasma stability is mitigated by a rapid accumulation of surface charges on the electrodes, thus allowing the atmospheric DBD to retain their character as a glow discharge. At certain values of the applied voltage, a highly complex phenomenon of period multiplication is observed in which the period of the discharge current is three times that of the applied voltage. This suggests that nonequilibrium atmospheric DBD may support evolution patterns that are quasiperiodic or even chaotic. These complex dynamic behaviors are likely to be critical to a full understanding of plasma stability of nonequilibrium atmospheric discharges and to the development of their instability control strategies. (C) 2006 American Institute of Physics. [DOI: 10.1063/1.2345463]
\end{abstract}

\section{INTRODUCTION}

Spatially diffuse and nonequilibrium dielectric-barrier discharges (DBDs) generated at atmospheric pressure have been a subject of active research because of their widespread use in many processing applications including surface modification, pollution control, and sterilization. ${ }^{1-5}$ Distinctly different from the filamentary mode to which atmospheric DBDs used to be associated, their glow mode allows them to be sustained at a gas temperature as low as room temperature. ${ }^{2}$ Essentially, nonequilibrium atmospheric DBDs are capacitively coupled glow discharges and they represent the most common group in the family of atmospheric pressure glow discharges (APGDs). Over the past five years, considerable advance has been made both in their scientific understanding and in their technological capability, benefited from a series of systematic studies of plasma dynamics both theoretically ${ }^{6-9}$ and experimentally. ${ }^{10-13}$ It is widely recognized that an in-depth understanding of plasma dynamic behaviors of atmospheric DBDs is likely to be indispensable to their future development.

Nonequilibrium atmospheric DBDs are a typical example of spatially extendable and dissipative systems with strong nonlinearity in both spatial and temporal dimensions. This is reflected in their complex spatiotemporal behaviors. For example, nonequilibrium atmospheric DBDs are known to possess a spatial structure consisting of a sheath region of

${ }^{\text {a)} E l e c t r o n i c ~ m a i l: ~ w a n g d e z @ d l u t . e d u . c n ~}$ high electric field, a bulk-plasma region of near neutrality, and several intermediate regions between the sheath region and the bulk-plasma region. ${ }^{4,6}$ This mode structure is very similar to that of low-pressure glow discharges. Other distinct spatial structures of atmospheric DBDs have also been observed, including transitions between filamentary and glow modes, ${ }^{14,15}$ self-organized discharge filaments and patterns, ${ }^{15,16}$ and striation. ${ }^{17,18}$ Similar complexity also exists in the temporal characteristics of nonequilibrium atmospheric DBDs. Early understanding of atmospheric DBDs was that their discharge events can be characterized by a single discharge current pulse of several microseconds every half cycle of the applied voltage. ${ }^{4,6,7}$ Subsequent experimental and computational studies have suggested that it is also possible to obtain several discharge current pulses every half voltage cycle. ${ }^{8-10}$ This suggests that the discharge system remains spatially homogeneous while it undergoes periodic temporal variations. In nonlinear science, such periodic temporal variation is generally known as oscillation. It is known that the oscillation is a common temporal feature of systems maintained far from the system equilibrium and that this temporal oscillation is often caused by Hopf bifurcation. ${ }^{19}$ Since atmospheric gas discharges have a tendency for glowto-arc transition, it is particularly desirable to distinguish oscillations associated with stable plasma operation from those that could lead to irreversible plasma instability and arcing.

This paper reports a theoretical study of complex dynamic behaviors in nonequilibrium atmospheric DBDs and 
how such temporal characteristics may impact on plasma stability. It is partly motivated by the success of using the current-voltage characteristics to identify glow modes in radio-frequency APGDs (Refs. 20 and 21) and subsequent correlation of mode characteristics to their plasma stability. ${ }^{22}$ This indicates that the current-voltage relationship provides a valuable signature of plasma dynamic behaviors in nonequilibrium atmospheric DBDs. Experimental evidence so far suggests that nonequilibrium atmospheric DBDs in their usual mode of one current pulse every half cycle are most likely to have the same physics roots as those with greater complexity of distinct spatiotemporal patterns. ${ }^{15}$ Therefore, our study will be based on a one-dimensional fluid model previously developed for nonequilibrium atmospheric DBDs. ${ }^{4,6-8,23}$

\section{DESCRIPTION OF THE PLASMA MODEL}

We consider a nonequilibrium atmospheric gas discharge generated and sustained between two dielectrically insulated parallel-plate electrodes. In most DBD experiments, the electrode gap size is much smaller than the width of the electrodes and so we assume that the plasma dynamics can be approximated reliably with a one-dimensional description. While spatial structures such as the sheath region may require more sophisticated electron-kinetics models, ${ }^{24,25}$ the currently prevailing hydrodynamic model is sufficiently accurate to study temporal behaviors. ${ }^{7,8,23}$ As a result, the diffusion-drift approximation is used and dynamical behaviors of all charged particles are described by their continuity equations. This description is subjected to a knowledge of the electric field between the two electrodes and the latter is determined by the Poisson equation. Hence in the onedimensional limit, the basic governing equations for atmospheric DBDs are described as

$$
\begin{aligned}
& \frac{\partial n_{e}}{\partial t}+\frac{\partial j_{e}}{\partial x}=S, \\
& \frac{\partial n_{i}}{\partial t}+\frac{\partial j_{i}}{\partial x}=S, \\
& j_{e}=-\mu_{e} E n_{e}-D_{e} \frac{\partial n_{e}}{\partial x}, \\
& j_{i}=+\mu_{i} E n_{i}-D_{i} \frac{\partial n_{i}}{\partial x},
\end{aligned}
$$

where the indices $e$ and $i$ correspond to electrons and ions, respectively, and $S$ is the source term. $n, j$, and $E$ are the particle densities, the flux densities, and the electric field, respectively, whereas $D$ and $\mu$ denote the mobility and diffusion coefficient. To focus on the most essential plasma properties, we consider only direct ionization by electron impact and recombination in the bulk of the gas gap. Plasma chemistry of interest to a particular DBD experiment is outside the scope of the current study and will be considered in a future note. With the above consideration, the source term takes the form of

$$
S=A p \mu_{e} E n_{e} e^{-B p /|E|}-\beta n_{e} n_{i},
$$

where $A$ and $B$ are constants related with the working gas, $p$ is the gas pressure, and $\beta$ is the recombination coefficient.

For the calculation of the electric field, the Poisson equation is usually solved together with the continuity equations. In the gas gap, the Poisson equation takes the form of

$$
\frac{\partial E}{\partial x}=\frac{e}{\varepsilon_{0}}\left(n_{i}-n_{e}\right) .
$$

To develop the above equation further, time differentiation is performed to both sides of the above equation. By using the continuity equation for space charges to relate charged particle densities to flux densities, the above equation is reduced to $^{26}$

$$
\frac{\partial E}{\partial t}=\frac{e}{\varepsilon_{0}} j_{0}-\frac{e}{\varepsilon_{0}} j_{g},
$$

where $j_{0}$ is the total flux density and $j_{g}=j_{i}-j_{e}$ is the conduction flux density in the gas gap. The total flux density is a function of time only, a result of charge conservation. The conduction flux density is, on the other hand, a function of both time and space, as it depends on the local balance of creation and loss of charged particles within the gas gap. The electric field in the gas gap can be calculated from the above current balance equation as an alternative to the Poisson equation (6). A spatial integration of Eq. (7) across the gas gap leads to

$$
\frac{d V_{g}}{d t}=\frac{e}{\varepsilon_{0}} j_{0} d_{g}-\frac{e}{\varepsilon_{0}} \int_{0}^{d_{g}} j_{g} d x,
$$

where $V_{g}$ is the voltage across the gas gap having a gap distance of $d_{g}$. It is important to note that the spatial integral of $j_{g}$ describes the net change of charged particles due to ionization, recombination, and excitation in the gas gap, not the loss of charged particles through adsorption on the electrode surfaces. In other words, it is not zero. The voltage fall across the two dielectric layers can be obtained from the relationship $V_{s}=V(t)-V_{g}$, where $V(t)$ is the applied voltage. By treating the dielectric as a capacitor, the time derivation of $V_{s}$ can be represented as

$$
\frac{d V_{s}}{d t}=\frac{2 e}{\varepsilon_{0} \varepsilon_{r}} j_{0} d_{s},
$$

where $\varepsilon_{r}$ is the relative permittivity of the dielectric barriers covering the two electrodes and $d_{s}$ is the thickness of each of the two dielectric-barrier layers. Combining Eqs. (8) and (9), the total flux density can be obtained. It is composed of a displacement flux density and a conduction flux density and is given by

$$
j_{0}=\frac{\varepsilon_{0}}{e\left(d_{g}+2 d_{s} / \varepsilon_{r}\right)} \frac{d V(t)}{d t}+\frac{1}{d_{s}+2 d_{s} / \varepsilon_{r}} \int_{0}^{d_{g}} j_{g} d x .
$$

For simplicity, we introduce $d=d_{g}+2 d_{s} / \varepsilon_{r}$. This also enhances the applicability of our results as it allows a straightforward application to, for example, barrier-free APGDs (Ref. 21) for which $d=d_{g}$. If we assume a sinusoidal applied voltage of $V(t)=V_{a} \sin (2 \pi f t)$ with $V_{a}$ being its amplitude and 
$f$ being its frequency, the total flux density of charged particles can be expressed simply as

$$
j_{0}=\frac{2 \pi f \varepsilon_{0} V_{a}}{e d} \cos \varphi+\frac{1}{d} \int_{0}^{d_{g}} j_{g} d x,
$$

where $\varphi=2 \pi f t$ is the phase of the applied voltage and its time derivation is

$$
\frac{\partial \varphi}{\partial t}=2 \pi f
$$

The total surface charge density $q$ deposited on the dielectric-barrier layers affects the dynamics of the gas plasma system and can be described by

$$
\frac{\partial q}{\partial t}=j_{g b}
$$

where $j_{g b}$ is the current density in the gas gap but immediately next to the dielectric barrier. The electric field produced by the surface charges can be estimated from $E_{q}=q / \varepsilon_{0}$ according to Gauss's theorem. Boundary conditions must be specified before the above equations can be solved. Secondary electron emission from the instantaneous cathode is considered here for ion bombardment alone, and therefore the electron current density leaving the cathode is given by $\gamma j_{i b}$, where $\gamma$ is the secondary electron emission coefficient and $j_{i b}$ is the ion current density at the instantaneous cathode surface.

The above equations can be further simplified with the following normalized quantities. ${ }^{26,27}$

$$
\begin{aligned}
& X_{0}=1 / A p, \quad E_{0}=B p, \quad V_{0}=E_{0} X_{0}, \\
& t_{0}=X_{0} / \mu_{e} E_{0}, \quad n_{0}=\varepsilon_{0} E_{0} / e X_{0} .
\end{aligned}
$$

So, we can deduce the dimensionless distance, time, electric field, voltage, and particle densities by changing the scales as follows:

$$
\begin{aligned}
& l=x / X_{0}, \quad \varepsilon=E / E_{0}, \quad U=V / V_{0}, \\
& \tau=t / t_{0}, \quad \rho_{e}=n_{e} / n_{0}, \quad \rho_{i}=n_{i} / n_{0} .
\end{aligned}
$$

With the above normalized variables, Eqs. (3) and (4) can be rewritten as follows:

$$
\begin{aligned}
& j_{i}=\mu_{e} E_{0} n_{0}\left(\mu \varepsilon \rho_{i}-\frac{\mu T_{i}}{V_{0}} \frac{\partial \rho_{i}}{\partial l}\right), \\
& j_{e}=\mu_{e} E_{0} n_{0}\left(-\varepsilon \rho_{e}-\frac{T_{e}}{V_{0}} \frac{\partial \rho_{e}}{\partial l}\right),
\end{aligned}
$$

where $\mu=\mu_{i} / \mu_{e}$ and $T_{i}$ and $T_{e}$ denote, respectively, the ion and electron temperatures. In deriving the above equations, we have used $D_{i}=T_{i} \mu_{i}$ and $D_{e}=T_{e} \mu_{e}$ according to Einstein's relation. For nonequilibrium atmospheric plasmas in general, the ion temperature is much lower than the electron temperature, namely, $T_{i} \ll T_{e}$. Using $\mu_{e} E_{0} n_{0}$ to divide into both sides of Eqs. (13) and (14), we introduce the dimensionless ion and electron current densities $J_{i}$ and $J_{e}$ as follows:

$$
\begin{aligned}
& J_{i}=\mu \varepsilon \rho_{i}-\frac{\mu T_{i}}{V_{0}} \frac{\partial \rho_{i}}{\partial l}, \\
& J_{e}=-\varepsilon \rho_{e}-\frac{T_{e}}{V_{0}} \frac{\partial \rho_{e}}{\partial l} .
\end{aligned}
$$

As a result, the dimensionless conduction current density in the gas gap is $J_{g}=J_{i}-J_{e}$. The continuity equations for electrons and ions now take the form of

$$
\begin{gathered}
\frac{\partial \rho_{e}}{\partial \tau}+\frac{\partial J_{e}}{\partial l}=\varepsilon \rho_{e} e^{-1 /|\varepsilon|}-\bar{\beta} \rho_{e} \rho_{i}, \\
\frac{\partial \rho_{i}}{\partial \tau}+\frac{\partial J_{i}}{\partial l}=\varepsilon \rho_{e} e^{-1 /|\varepsilon|}-\bar{\beta} \rho_{e} \rho_{i},
\end{gathered}
$$

where $\bar{\beta}=\varepsilon_{0} \beta /\left(e \mu_{e}\right)$ is the dimensionless recombination coefficient. Similarly, the total dimensionless discharge current density $J_{0}$, the dimensionless voltage over the gas gap $U_{g}$, and the dimensionless surface charge $Q=q / e \mu_{e} E_{0} n_{0} t_{0}$ can be described by the following equations:

$$
\begin{aligned}
& J_{0}=\frac{1}{L}\left[\frac{1}{V_{0}} \frac{d V(t)}{d \tau}+\int_{0}^{L_{g}} J_{g} d l\right], \\
& \frac{d U_{g}}{d \tau}=J_{0} L_{g}-\int_{0}^{L_{g}} J_{g} d l, \\
& \frac{d Q}{d \tau}=J_{g b},
\end{aligned}
$$

where $L=d / X_{0}$ and $L_{g}=d_{g} / X_{0}$. Consequently, the dynamic behaviors of nonequilibrium atmospheric DBDs are described by the above dimensionless equations and controlled by key system parameters, particularly $V_{a}, f$, and $L$. All these parameters can significantly influence plasma dynamics. For this study, we confine our discussion to the effect of $V_{a}$, the peak applied voltage. The above equations were discretized and solved with the Schartetter-Gummel scheme ${ }^{26,28}$ and the algorithm is detailed in a previous report. ${ }^{29}$

\section{RESULTS AND DISCUSSIONS}

For all numerical examples presented here, the working gas was helium at 760 torr and the frequency of the applied sinusoidal voltage was $10 \mathrm{kHz}$. The gas gap between the two dielectrically insulated electrodes was fixed at $2 \mathrm{~mm}$, and the two dielectric layers were identical with each other having a thickness of $1 \mathrm{~mm}$ and a relative permittivity of 7.6. The secondary electron emission coefficient $\gamma$ was set to 0.02 , whereas drift coefficients as well as reaction rate constants were taken from a recent DBD simulation study ${ }^{30}$ and the corresponding diffusion coefficients can be obtained by Einstein's relation.

As the applied voltage increased to be near but below the breakdown voltage of the gas gap, weak ionization events started to take place before the avalanche was reached. Under this prebreakdown condition, the total discharge current consists of mainly the displacement current. With a peak applied voltage of $1 \mathrm{kV}$, the temporal evolution of the dis- 

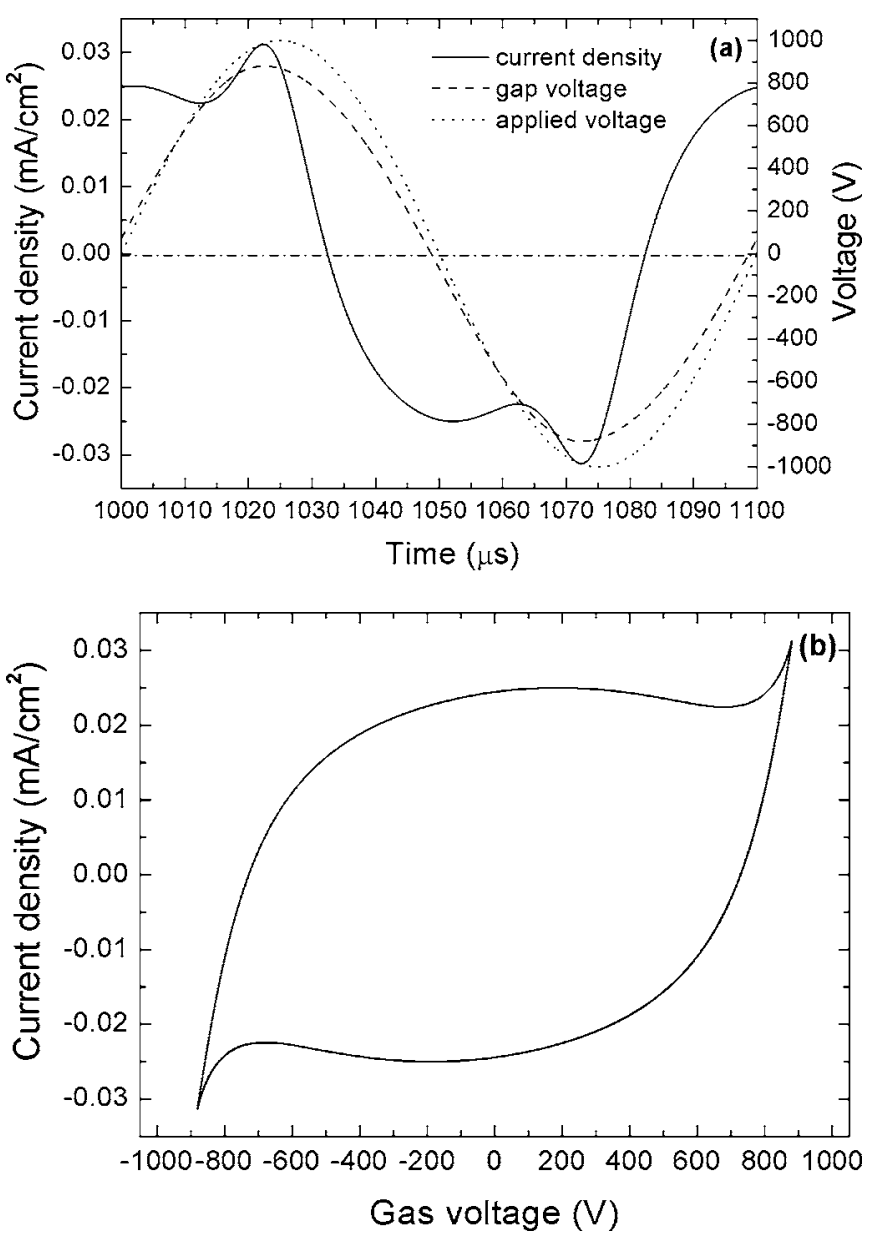

FIG. 1. Plasma dynamics under a prebreakdown condition having a peak applied voltage of $1.0 \mathrm{kV}$, described by (a) time traces of the discharge current density and the applied and gas voltages and (b) the relationship between the current density and the gas voltage.

charge current density is shown in Fig. 1(a) together with that of the gas voltage and the applied voltage. Because of the relatively small applied voltage, the discharge current density was always below $32 \mu \mathrm{A} / \mathrm{cm}^{2}$ and its wave form was that of a distorted sinusoidal. On the other hand, the gas voltage is seen in Fig. 1(b) to follow an approximately sinusoidal pattern. These features are different from the usual DBD pattern of one sharp current pulse every half cycle. ${ }^{4}$ To understand the basic current-voltage characteristics, the instantaneous discharge current density is plotted in Fig. 1(b) as a function of its corresponding instantaneous gas voltage. This current-voltage relationship is closed, suggesting that the plasma dynamics are essentially periodic under the prebreakdown condition of very low current densities. It is worth noting that the closed loop in Fig. 1(b) covers the entire range of the gas voltage from -900 to $900 \mathrm{~V}$. This is a direct result of the current pulse remaining large over the entire period of the applied voltage in Fig. 1(a). From the standpoint of nonlinear dynamical systems, the closed loop in Fig. 1(b) is a so-called limited cycle and indicates a periodic state of the discharge system. ${ }^{31}$ This is a simple state of periodicity, with which the dynamical behaviors of the discharge can be described completely by the discharge current and the gas voltage alone.
A significant feature of the current-voltage relationship in Fig. 1(b) is that it always has a positive differential conductivity. So the discharge current increases when the gas voltage increases, and vice versa. This implies that the growth of the discharge current can be easily limited if the electrical power input from the power supply to the plasma rig is capped. ${ }^{32}$ Hence by restricting the input power using simple power electronics techniques, nonequilibrium atmospheric DBDs with a positive differential conductivity can achieve very high stability. ${ }^{33}$

As the peak applied voltage increased to $1.5 \mathrm{kV}$, gas breakdown occurred and the classic DBD characteristic of one current pulse every half cycle was observed. This is shown in Fig. 2(a), similar to that previously reported. ${ }^{4,10}$ The width of the current pulse was about $2 \mu \mathrm{s}$, a fraction of the voltage period of $100 \mu \mathrm{s}$. The peak current was $1.75 \mathrm{~mA} / \mathrm{cm}^{2}$ now, some 60 times larger than that in Fig. 1(a). Also significantly changed was the relationship of the current density and the gas voltage as shown in Fig. 2(b). While this is again a closed pattern and so represents a repetitive temporal behavior from cycles to cycles, it consists of two individual closed loops interlinked by two lines of very low current densities. These two closed loops correspond to the two discharge current pulses in Fig. 2(a). They are confined to two small voltage ranges of $625-1070 \mathrm{~V}$ and from -625 to $-1070 \mathrm{~V}$, in which the magnitude of the discharge current reaches its maximum in Fig. 2(a). In other words, the size of the closed loops in Fig. 2(b) is determined directly by the voltage range in which the discharge current is most significant. This correlation is also true in the case of Fig. 1. Furthermore, it is worth mentioning that the area of the loops is proportional to dissipation power density.

It is interesting to note that each of the two closed loops in Fig. 2 can have either positive differential conductivity or negative differential conductivity. A negative differential conductivity implies that the discharge current can in principle grow without restriction even when the input power is capped. ${ }^{32}$ Hence with negative differential conductivity, nonequilibrium atmospheric DBDs become less stable. In Fig. 2(b), the atmospheric DBD is shown to acquire a negative differential conductivity when it evolves from point $a$ to point $b$. This evolution is accompanied with a rapid increase of the discharge current and a simultaneous reduction of the gas voltage. Theoretically this can lead to a glow-to-arc transition with unrestricted current growth. Yet the current growth is halted at point $b$, when the differential conductivity of the gas gap changes from being negative to positive. Relating it to Fig. 2(a), point $b$ is also halted when sufficient ionization-generated electrons reach one dielectric layer to alter the memory voltage, namely, the voltage falling on the dielectric barrier, ${ }^{4}$ and subsequently reduce the gas voltage to below the breakdown voltage. As a result, the production of new electron-ion pairs is prevented and the discharge current starts to reduce rapidly. So, although negative differential conductivity is induced in Fig. 2(b), the theoretical possibility of an unrestricted current growth is removed very effectively through depositing charged particles on the dielectric barriers in nonequilibrium atmospheric DBDs. The use of the dielectric layers controls the duration over which the gas 

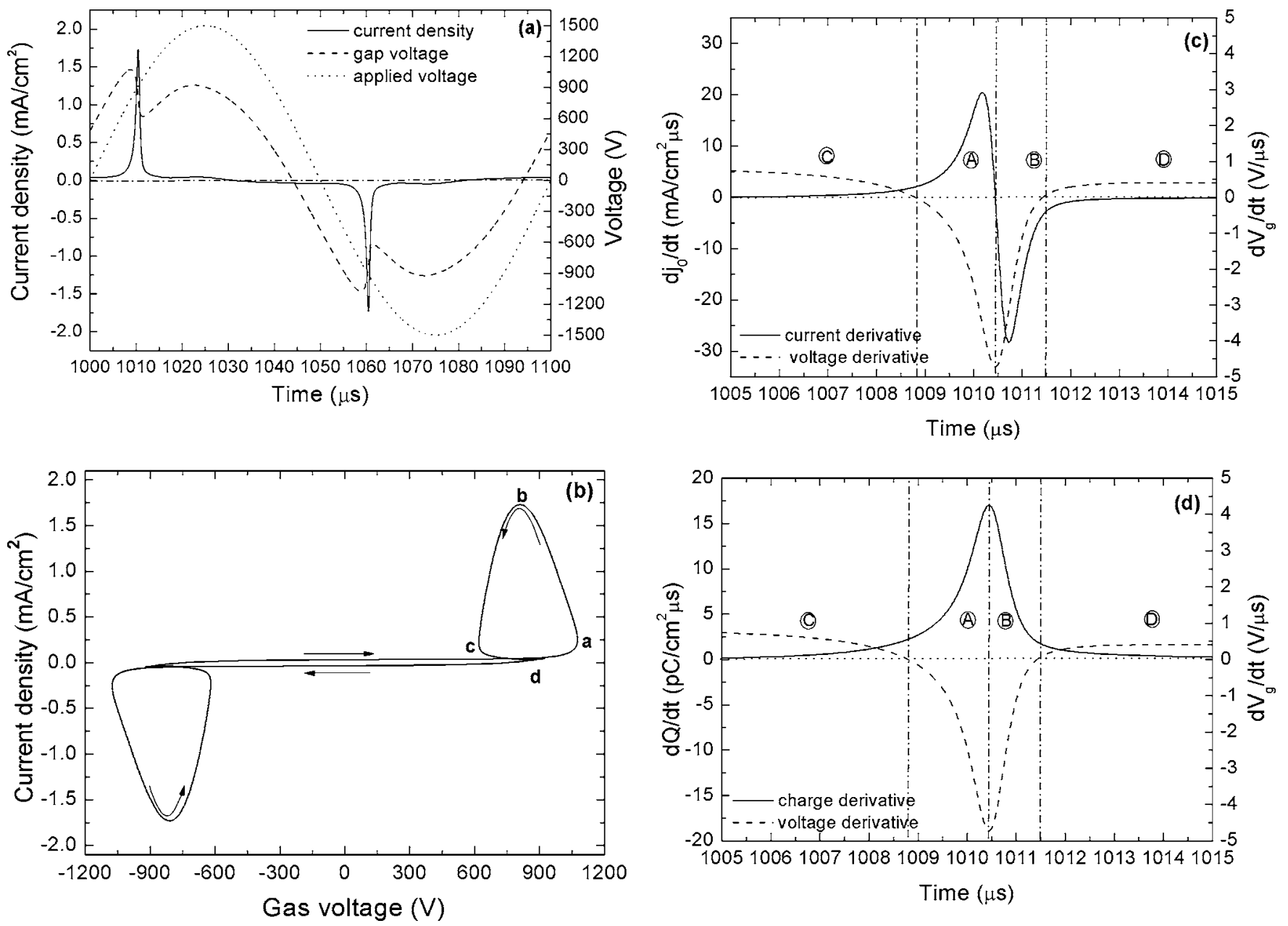

FIG. 2. Plasma dynamics at a peak applied voltage of $1.5 \mathrm{kV}$, described by (a) time traces of the discharge current density and the applied and gas voltages, (b) the relationship between the current density and the gas voltage, (c) temporal dependence of the time derivatives of the current density and the gas voltage, and (d) temporal dependence of the time derivatives of the surface charge density and the gas voltage.

voltage can exceed the breakdown voltage and as such suppress the glow-to-arc transition. In Fig. 2(b), the differential conductivity is negative also in the region from point $c$ to $d$. However, since the temporal trend of the discharge is from a high current at point $c$ to a low current point at point $d$, this region of negative differential conductivity does not present any danger for a glow-to-arc transition.

It is of interest to see clearly the temporal evolution of the differential conductivity. Since a direct calculation of $d U / d I$ leads to mathematical singularities when $d I / d t$ approaches to zero, we plot the time derivatives of the gas voltage and the current density in Fig. 2(c). It is evident that the breakdown avalanche occurs most significantly in the two regions marked as $A$ and $B$ where time derivatives of both the discharge current density and the gas voltage change considerably. In region $A$, the time derivative of the current density is always positive and that of the gas voltage is always negative. Hence, the discharge current increases while the gas voltage decreases, suggesting a negative differential conductivity. As the discharge evolves into region $B$, the gas voltage maintains a negative time derivative and so continues to reduce. However, the discharge current density stops to increase and starts to decrease now with a negative time derivative. The discharge plasma now has a positive differ- ential conductivity. Hence the boundary between region $A$ and region $B$ represents a transition point from negative differential conductivity to positive differential conductivity. This transition point corresponds to point $b$ in Fig. 2(b).

There are two additional regions in Fig. 2(c), both with small time derivatives of the discharge current and the gas voltage. Therefore, plasma stability in these two regions is unlikely to change significantly, even though region $C$ has positive differential conductivity and region $D$ has negative differential conductivity. Using the differential conductivity, it is possible to directly correlate the temporal behaviors of the atmospheric DBD to its plasma stability. Prior to a strong ionization event in region $C$, the discharge evolves with an increasing current density and a positive differential conductivity. Upon its entrance into region $A$, its gas voltage starts to decrease and its differential conductivity becomes negative, thus compromising the plasma stability. As the discharge current continues to grow, the differential conductivity decreases further and the atmospheric DBD becomes progressively less stable. The decline of the plasma stability is halted, however, after the atmospheric DBD enters into region $B$. Now with a positive differential conductivity, the discharge current starts to decrease while the gas voltage continues to decrease. Eventually when the plasma enters 
region $D$, both the discharge current and the gas voltage are so small that they do not affect plasma stability even though the differential conductivity is again negative. The direct correlation of plasma stability to differential conductivity is useful as a tool to electrically monitor APGD stability and as an implement mechanism for any instability control strategy, both achievable using traditional power electronics techniques.

It is possible to show analytically the association of negative differential conductivity to the short period of very large discharge current. To demonstrate this, we reformulate Eqs. (19) and (20) and obtain the time derivative of the gas voltage as follows:

$$
\frac{d U_{g}}{d \tau}=\frac{L_{g}}{L} \frac{1}{V_{0}} \frac{d V(t)}{d \tau}-\frac{2 \varepsilon_{r} L_{b}}{L} \int_{0}^{L} J_{g} d l .
$$

It is evident that the first term on the right hand side of Eq. (22) represents the displacement current and the second term represents the conduction current. When the plasma system is away from the state of electron avalanche, there are few space charges in the gas gap and the contribution from the conduction current is very small. So, Eq. (22) can be written as

$$
\frac{d U_{g}}{d \tau} \approx \frac{L_{g}}{L} \frac{1}{V_{0}} \frac{d V(t)}{d \tau}
$$

As a result, the temporal change of the gas voltage depends largely on the displacement current. They increase together with a positive differential conductivity. As the plasma system evolves towards the state of electron avalanche, more and more electron-ion pairs are produced to result in substantially more space charges in the gas gap. This leads to a rapid increase in the conduction current: thus the second term on the right hand side of Eq. (22), namely, the conduction current, becomes dominant. Consequently Eq. (22) can be approximated as

$$
\frac{d U_{g}}{d \tau} \approx-\frac{2 \varepsilon_{r} L_{b}}{L} \int_{0}^{L} J_{g} d l .
$$

Hence $d U_{g} / d t$ is always negative when the current density is positive and dominated by the conduction current. This suggests negative differential conductivity while conduction current is increasing. As the gas voltage continues to decrease and electrons drifting towards the instantaneous anode become adsorbed there, the electric field in the gas gap reduces. This leads to an increasing reduction in the level of gas ionization and in turn it reduces the contribution of the conduction current. Eventually Eq. (24) reverts back to Eq. (23) and the differential conductivity becomes positive again.

The dynamic evolution of the gas voltage can be seen more clearly with the surface change on the electrodes. Figure 2(d) plots the time derivatives of the surface charge density and the gas voltage, both as a function of time. As the plasma system approaches the electron avalanche in region $A$ from region $C$, more and more electron-ion pairs are produced resulting in increase in space charges in the gas gap, and then the surface charges deposited on the dielectric barrier increase. The additional electric fields produced by the surface charges reduce the total fields in the gas gap and hence the gas voltage. While this suppresses gas ionization in gas gap, the discharge current continues to increase in region $A$ because of the large number of space charges available in the gas gap. With the discharge current carrying a large quantity of charged particles to the two electrodes, the time derivative of the surface charges remains positive and increases rapidly as shown in Fig. 2(d). Eventually the depletion of available charged particles in the gas gap combines with a reducing gas voltage to halt the growth of the discharge current and hence that of the surface charges. This occurs at the boundary between regions $A$ and $B$. Therefore in dielectricbarrier discharges, a reduction in the discharge current follows that of the gas voltage but at a delayed point in time. The differential conductivity of the ionized gas is negative during this delayed period of time, and it becomes positive once sufficient charged particles are adsorbed on the electrodes to suppress current growth.

It is worth revisiting Fig. 2(b) where the relationship between the discharge current and the gas voltage consists of two linked and closed loops. From the standpoint of nonlinear dynamical systems, ${ }^{31}$ this is again a limited cycle because of the pattern being closed and as such the discharge dynamics are periodic. However, the closed pattern has two intersection points and without the aid of arrows the direction of subsequent dynamical evolution is uncertain. The appearance of intersections in Fig. 2(b) indicates that a full description of plasma dynamics requires a third quantity in addition to the discharge current and the gas voltage. From the foregoing discussion of Fig. 2(d), surface charges appear to be an important physical quantity. To this end, plasma dynamics are represented in a three-dimensional space of the surface charge density, the discharge current, and the gas voltage in Fig. 3. It is evident that the trajectory of the plasma dynamics is closed without any intersection and is therefore a threedimensional limited cycle. ${ }^{31}$ Hence by increasing the magnitude of the applied voltage, the two-dimensional limited cycle of Fig. 1(b) evolves into the three-dimensional limited cycle of Fig. 3. This represents a significant change in the character and periodicity of the plasma system or bifurcation as discussed comprehensively for nonlinear dynamical systems. $^{31,32}$

The general pattern of the three-dimensional limited cycle in Fig. 3 remains unchanged regardless of the applied voltage, as long as the classic temporal feature in Fig. 2(a) of one current pulse per half voltage cycle is retained. However, when the amplitude of the applied voltage increases further, it is possible to obtain two or more discharge current pulses per half voltage cycle. ${ }^{9}$ An example of such scenarios is shown for the case of $V_{a}=2.0 \mathrm{kV}$ in Fig. 4(a) where four closed loops are evident. The four loops correspond to two discharge current pulses in the first half cycle and further two current pulses in the second half cycle of the applied voltage. Similar to that in Fig. 2(b), these loops acquire both positive and negative differential conductivities. Temporal variations of the time derivatives of the discharge current, and the gas voltage are shown in Fig. 4(b), similar to those in Fig. 2(c). The trajectory of plasma dynamics is again described three dimensionally in Fig. 4(c) by means of the surface charge 


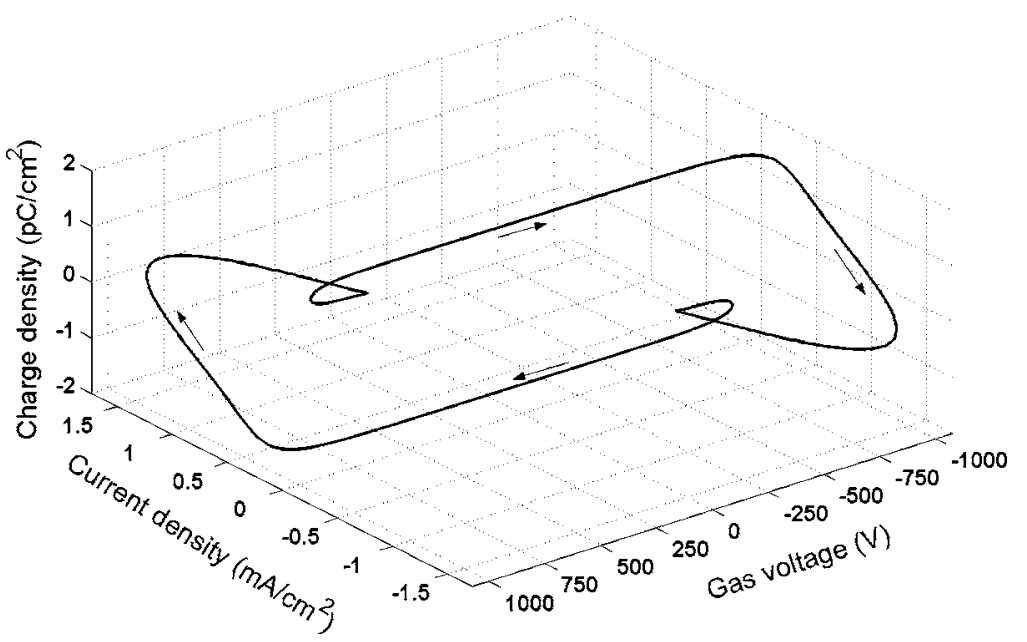

FIG. 3. Dynamic evolution trajectory of the atmospheric DBDs in the three-dimensional phase space of the gas voltage, the current density, and the surface charge density, with the peak applied voltage fixed at $1.5 \mathrm{kV}$.

density, the discharge current and the gas voltage. Similar to that in Fig. 3, this three-dimensional limited cycle is closed without any intersection. Therefore, the plasma dynamics remain periodic even though more than one significant ionization event is induced during each half voltage cycle. It is worth noting that the three-dimensional limited cycle of Fig. 4(c) has two symmetric spiral sections for which plasma evolution occurs largely along the direction of the charge-
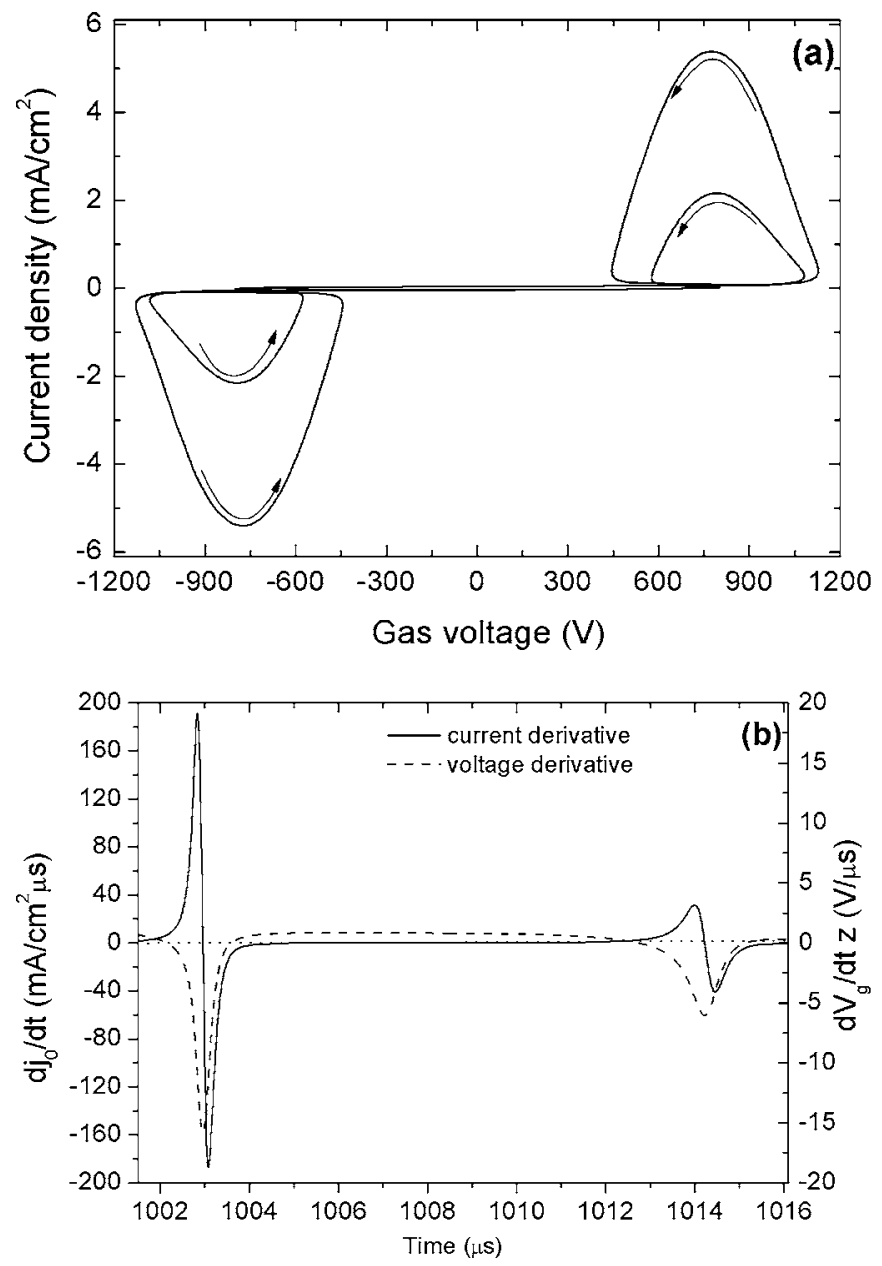

density axis. Each of these two spiral sections has two convolutions, corresponding to two current pulses in each half voltage cycle. This contrasts that in Fig. 3 where there is no spiral section.

With an increase in the peak applied voltage from 1.5 to $2.0 \mathrm{kV}$, plasma dynamics undergo an abrupt change in its three-dimensional limited cycle from Fig. 3 to Fig. 4(c). This represents a bifurcation. In fact, additional bifurcation

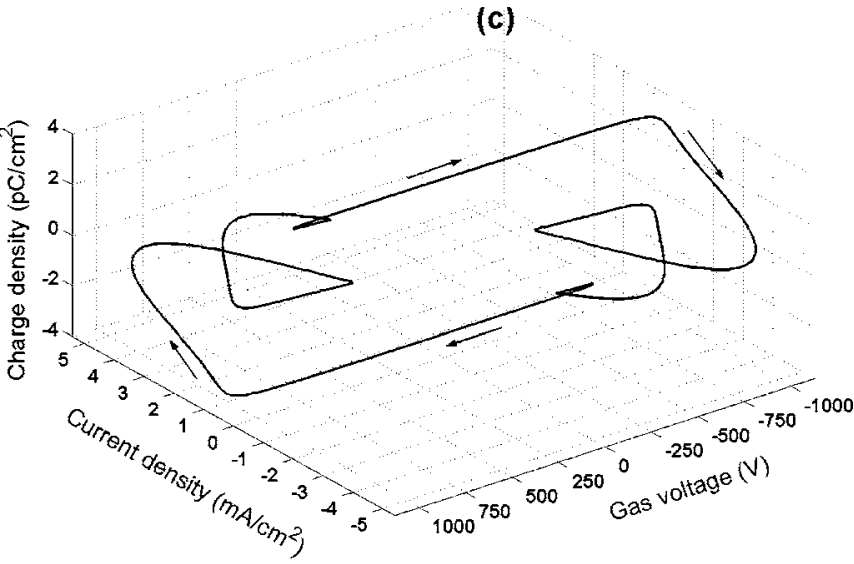

FIG. 4. Plasma dynamics at a peak applied voltage of $2.0 \mathrm{kV}$, described by (a) the relationship between the current density and the gas voltage, (b) temporal dependence of the time derivatives of the current density and the gas voltage, and (c) the three-dimensional trajectory in the voltage-current-charge phase space. 


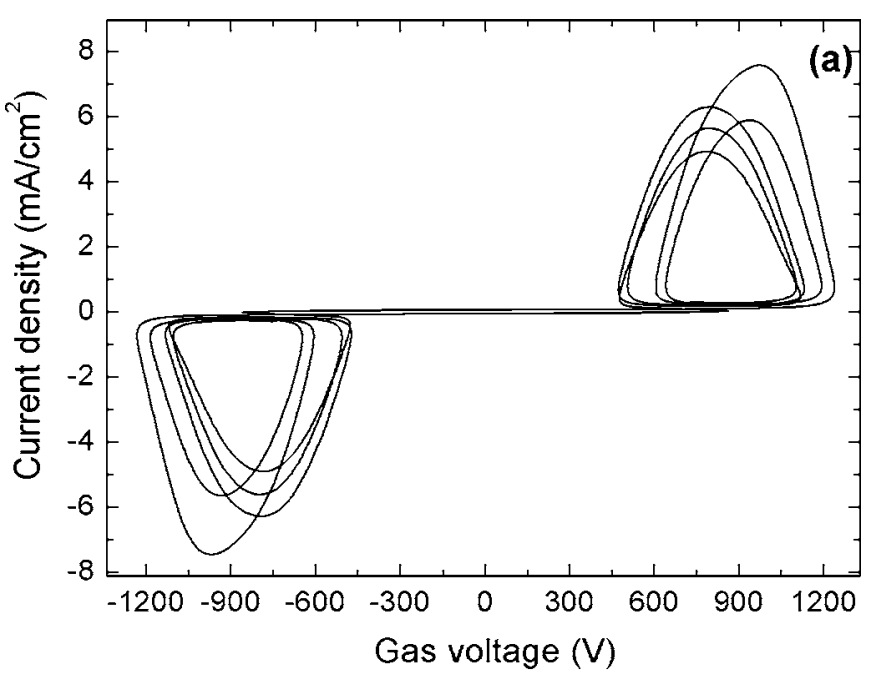

(b)

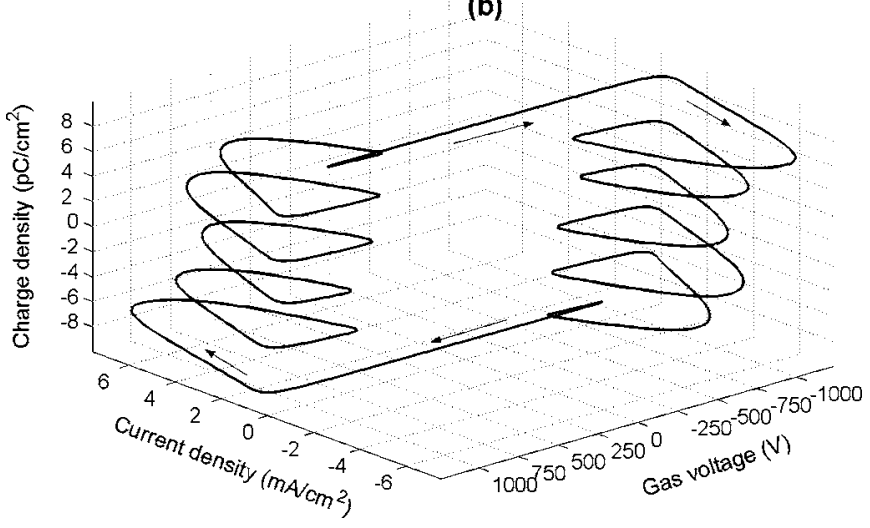

FIG. 5. Plasma dynamics at a peak applied voltage of $4.0 \mathrm{kV}$, described by (a) the relationship between the current density and the gas voltage and (b) the three-dimensional trajectory in the voltage-current-charge phase space.

events can be induced if the applied voltage is increased still further. Figure 5 shows (a) the relationship of the gas voltage and the discharge current density and (b) its corresponding three-dimensional limited cycle when the peak applied voltage is increased to $4.0 \mathrm{kV}$. There are now five current pulses per half voltage cycle in the time traces of voltages and currents (not shown), corresponding to the two sets of five loops in Fig. 5(a) and to the two symmetric spiral sections, in the direction of the charge-density axis and each with five convolutions, of the three-dimensional limited cycle in Fig. 5(b). It is conceivable that the number of the current pulses per half voltage cycle increases from 2, through 3 and 4, to 5 as the peak applied voltage increases from 2.0 to $4.0 \mathrm{kV}$. Therefore in the wide range of the applied voltage from 1.5 to $4.0 \mathrm{kV}$, the atmospheric DBD evolves through a sequence of dynamic phases ranging from that characterized by the simplistic two-dimensional limited cycle in Fig. 1(b), through that characterized by the simplest three-dimensional limited cycle having no spiral section in Fig. 3, and finally to the complex three-dimensional limited cycles in Figs. 4(c) and 5(b) having two symmetric spiral sections of increasing convolutions. Preliminary results of our two-dimensional simulation $^{34}$ indicate that the overall spatial appearance of the discharge plasma remains largely unchanged. Hence, the

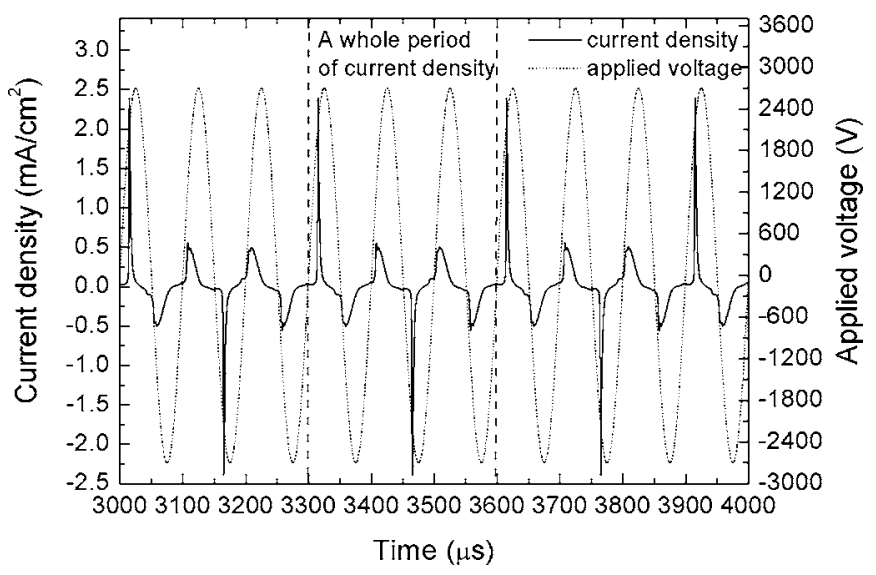

FIG. 6. Observation of a period 3 structure as demonstrated by time traces of the discharge current density and the applied voltage. The peak applied voltage is $2.7 \mathrm{kV}$, the gas gap is $10 \mathrm{~mm}$, and the secondary electron emission coefficient is 0.01 .

dynamic evolution of the atmospheric DBDs is through a sequence of bifurcation changes-phase changes in the time domain but not in the space domain.

From the above numerical examples as well as reported experimental data, the dynamic behaviors of nonequilibrium atmospheric DBDs appear to be of a periodic character. However, quasiperiodic and chaotic behaviors have been observed in other plasma systems ${ }^{35,36}$ and we have no reasonable justification to exclude the existence of such nonperiodic trajectories in nonequilibrium atmospheric plasmas. While a comprehensive discussion is outside the scope of this work, we have indeed observed period multiplication from our numerical simulation. Figure 6 shows an example of period multiplication in which the period of the discharge current is three times that of the applied voltage when the peak applied voltage is $2.7 \mathrm{kV}$ and a gap distance of $10 \mathrm{~mm}$. In other words, the plasma system of Fig. 6 exhibits a socalled period 3 structure. According to the seminal work of $\mathrm{Li}$ and Yorke, ${ }^{37}$ a period 3 structure may be indicative of chaotic behaviors. This is significant as it highlights a probability of quasiperiodic or even chaotic dynamic behaviors in nonequilibrium atmospheric plasmas. While this must be addressed in a separate note, its implication is profound for stability analysis of nonequilibrium atmospheric plasmas and for the development of their instability control strategies.

\section{CONCLUSION}

In this paper, the complex dynamics behaviors of an atmospheric DBD were studied with a one-dimensional fluid model. In addition to the time traces of the discharge currents and applied and gas voltages, a three-dimensional phase space of gas voltage, discharge current density, and electrode-surface charge density was used as the main analysis tool to unravel and understand the complex dynamic behaviors of atmospheric DBDs and their evolution as the applied voltage increased from prebreakdown to many times above the breakdown voltage. It was shown that the dynamic evolution of the atmospheric DBDs passed through a sequence of phases ranging from a simplistic two-dimensional limited cycle, through the simplest three-dimensional limited 
cycle, and to a complex three-dimensional limited cycle having two symmetric spiral sections of increasing convolutions. Above the breakdown voltage, the discharge plasma was shown to acquire negative differential conductivity and as a result its stability was compromised. This was, however, mitigated by a rapid accumulation of surface charges on the electrodes, thus allowing the atmospheric DBD to retain its character as a glow discharge. At certain values of the peak applied voltage, a highly complex phenomenon of period multiplication was observed in which the period of the discharge current is three times that of the applied voltage. This suggests that nonequilibrium atmospheric DBDs may support evolution patterns that are quasiperiodic or even chaotic. These complex dynamic behaviors are likely to be critical to a full understanding of plasma stability of nonequilibrium atmospheric plasmas and to the development of their instability control strategies.

\section{ACKNOWLEDGMENT}

This work was supported by the National Natural Science Foundation of China under Grant Nos. 50528707 and 50537020 .

${ }^{1}$ B. Eliasson and U. Kogelschatz, IEEE Trans. Plasma Sci. 19, 1063 (1991).

${ }^{2}$ E. E. Kunhardt, IEEE Trans. Plasma Sci. 28, 189 (2000).

${ }^{3}$ T. Yokoyama, M. Kogoma, T. Moriwaki, and S. Okazaki, J. Phys. D 23 , 1125 (1990).

${ }^{4}$ F. Massines, A. Rabehi, Ph. Decomps, R. B. Gadri, P. Segur, and C. Mayoux, J. Appl. Phys. 83, 2950 (1998).

${ }^{5}$ T. C. Montie, K. Kelly-Wintenberg, and J. R. Roth, IEEE Trans. Plasma Sci. 28, 41 (2000)

${ }^{6}$ Yu. B. Golubovskii, V. A. Maiorov, J. Behnke, and J. F. Behnke, J. Phys. D 35, 751 (2002).

${ }^{7}$ M. G. Kong and X. T. Deng, IEEE Trans. Plasma Sci. 31, 7 (2003).

${ }^{8}$ Yu. B. Gloubovskii, V. A. Maiorov, J. Behnke, and J. F. Behnke, J. Phys. D 36, 39 (2003).
${ }^{9}$ I. Radu, R. Bartnikas, and M. R. Wertheimer, IEEE Trans. Plasma Sci. 31, 1363 (2003).

${ }^{10}$ Y. S. Akishev, A. V. Dem'yanov, V. B. Karal'nik, M. V. Pan'kin, and N. I. Trushkin, Plasma Phys. Rep. 27, 176 (2001).

${ }^{11}$ N. Gherardi and F. Massines, IEEE Trans. Plasma Sci. 29, 536 (2001).

${ }^{12} \mathrm{C}$. Anderson, M. Hur, P. Zhang, L. Mangolini, and U. Kortshagen, J. Appl. Phys. 96, 1835 (2004)

${ }^{13}$ G. Nersisyan, T. Morrow, and W. G. Graham, Appl. Phys. Lett. 85, 1487 (2004).

${ }^{14}$ U. Kogelschatz, IEEE Trans. Plasma Sci. 30, 1400 (2002).

${ }^{15}$ I. Radu, R. Bartnikas, and M. R. Wertheimer, IEEE Trans. Plasma Sci. 31, 411 (2003)

${ }^{16}$ L. Dong, Z. Mao, Z. Yin, and J. Ran, Appl. Phys. Lett. 84, 5142 (2004).

${ }^{17}$ Y. Yang, J. J. Shi, J. E. Harry, J. Proctor, C. P. Garner, and M. G. Kong, IEEE Trans. Plasma Sci. 33, 302 (2005).

${ }^{18}$ F. Iza and J. A. Hopwood, IEEE Trans. Plasma Sci. 33, 306 (2005).

${ }^{19}$ C. Strumpel, Y. A. Astrov, and H.-G. Purwins, Phys. Rev. E 62, 4889 (2000).

${ }^{20}$ J. J. Shi, X. T. Deng, R. Hall, J. D. Punnett, and M. G. Kong, J. Appl. Phys. 94, 6303 (2003).

${ }^{21}$ J. J. Shi and M. G. Kong, IEEE Trans. Plasma Sci. 33, 624 (2005).

${ }^{22}$ J. J. Shi and M. G. Kong, Appl. Phys. Lett. 86, 091501 (2005).

${ }^{23}$ Y. T. Zhang, D. Z. Wang, and Y. H. Wang, Phys. Plasmas 12, 103508 (2005).

${ }^{24}$ X. M. Zhu and M. G. Kong, J. Appl. Phys. 97, 083301 (2005).

${ }^{25}$ J. J. Zhu and M. G. Kong, J. Appl. Phys. 97, 023306 (2005).

${ }^{26}$ A. A. Kulikovsky, J. Phys. D 27, 2556 (1994).

${ }^{27}$ D. D. Sijacic and U. Ebert, Phys. Rev. E 66, 0664101 (2002).

${ }^{28}$ A. A. Kulikovsky, J. Comput. Phys. 119, 149 (1995).

${ }^{29}$ Y. T. Zhang, D. Z. Wang, Y. H. Wang, and C. S. Liu, Chin. Phys. Lett. 22, 171 (2005).

${ }^{30}$ L. Mangolini, C. Anderson, J. Heberlein, and U. Kortshagen, J. Phys. D 37, 1021 (2004)

${ }^{31}$ E. Ott, Chaos in Dynamical Systems (Cambridge University Press, Cambridge, 2002).

${ }^{32}$ H. Haken, Advanced Synergetics (Springer-Verlag, Berlin, 1983).

${ }^{33}$ J. J. Shi and M. G. Kong, Appl. Phys. Lett. 87, 201501 (2005).

${ }^{34}$ Y. T. Zhang, D. Z. Wang, and M. G. Kong, J. Appl. Phys. 98, 113308 (2005).

${ }^{35}$ J. Qin, L. Wang, D. P. Yuan, P. Gao, and B. Z. Zhang, Phys. Rev. Lett. 63, 163 (1989)

${ }^{36}$ D. D. Sijacic, U. Ebert, and I. Rafatov, Phys. Rev. E 70, 0562201 (2004).

${ }^{37}$ T. Y. Li and J. A. Yorke, Am. Math. Monthly 82, 985 (1975). 